INRA Prod. Anim., 2004, 17 (1), 59-68

\section{GUEMENE, J.-M. FAURE}

INRA, Station de Recherches Avicoles, F-37380 Nouzilly

Courriel :

Daniel.Guemene@tours.inra.fr

\section{Productions} avicoles, bien-être et législation européenne
La prise en compte de la nécessité de protéger les animaux de rente n'est pas récente puisqu'un premier texte, dit "loi Grammont", s'y référant a été adopté en France en 1850. La perception de ce concept de protection animale a toutefois évolué vers celui de bien-être animal. Parallèlement, les modes de production dans le secteur animal ont subi une profonde mutation, notamment pour les filières avicoles de production intensives, et la question du respect du bien-être se pose avec une acuité accrue. Le législateur répond à cette "demande sociale" en faisant évoluer la législation existante et par l'élaboration et l'adoption d'une réglementation spécifique. Ainsi, le comité permanent du Conseil de l'Europe a adopté, en 1976, une convention concernant les différentes espèces domestiques. En 1998, l'Union Européenne a également adopté une directive généraliste. Plus récemment, l'Union Européenne a adopté une seconde directive concernant spécifiquement la poule pondeuse

\section{Résumé}

En Europe, l'élaboration de la législation concernant le bien-être animal est le fait de deux organisations qui sont le Conseil de l'Europe et l'Union Européenne. Une convention générale concernant toutes les espèces domestiques et par conséquent les oiseaux (1976) ainsi qu'une directive ayant la même portée $(98 / 58 / \mathrm{CE}, 1998)$ ont été adoptées, respectivement, par le comité permanent de la Convention européenne (Conseil de l'Europe) et l'Union Européenne. Par ailleurs, l'Union Européenne a adopté une seconde directive spécifique, concernant la poule pondeuse (Gallus gallus) (1999). Des rapports scientifiques concernant respectivement les poules pondeuses (19921996), la production de foie gras (1998) et le poulet de chair (2000) ont été rédigés à la demande du comité scientifique vétérinaire (CSV) de l'Union Européenne, remplacé aujourd'hui par l'Autorité Européenne de Sécurité des Aliments (AESA). Parallèlement, le comité permanent du Conseil de l'Europe a adopté six recommandations spécifiques concernant l'espèce Gallus (1986), les ratites (1997), les palmipèdes (trois recommandations en 1999) et la dinde (2001). Conformément à la convention de 1976, toutes ces recommandations doivent êtres révisées dans un délai de cinq ans après leur adoption et peuvent êtres éventuellement amendées. En outre, selon la directive 98/58/CE (Union Européenne 1998), chaque recommandation adoptée doit être ensuite transcrite en droit national par les états membres. En tout état de cause, ces textes nationaux ne peuvent qu'être plus restrictifs.
(Gallus gallus). Parallèlement, le comité permanent du Conseil de l'Europe a adopté six recommandations spécifiques concernant différentes espèces d'oiseaux domestiques. Dans le présent document, après avoir brièvement défini la notion de bien-être et évoqué la législation nationale, nous rapporterons successivement les processus d'élaboration de la réglementation européenne, les instances européennes concernées et, enfin, le contenu des textes s'adressant aux différentes filières avicoles et les conséquences potentielles en termes de conduite d'élevage et de systèmes de production.

\section{1 / Qu'est-ce que le bien-être animal pour le législateur?}

En préambule, il n'est sans doute pas inutile de préciser que la définition du terme bienêtre animal ne figure dans aucun texte officiel. On comprendra que dans ce contexte, son interprétation peut différer sensiblement en fonction de l'interlocuteur, selon qu'il s'agisse d'un législateur, d'un scientifique, d'un consommateur, d'un éleveur ou d'un protecteur des animaux. Pour le législateur, "protection animale" et "bien-être animal" sont en fait des termes équivalents qui sont utilisés indifféremment dans les textes officiels. Un groupe de parlementaires britanniques en a donné une définition (Rapport Brambell 1965 , cité dans Dawkins 1983) qui a ensuite été reprise par une organisation para-gouvernementale britannique, le FAWC (Farm Animal Welfare Council). Cette définition plurifactorielle est souvent résumée par la notion du respect de cinq libertés fondamentales ou Five freedom. Elle prend en compte le respect de besoins physiologiques (alimentation), environnementaux (confort de l'animal), sanitaires (maladies, blessures), psychologiques (peur, anxiété) et comportementaux. Une définition plus récente tient 
également compte du rôle actif que peut jouer l'animal et de sa capacité d'adaptation. L'animal doit alors pouvoir satisfaire ses besoins sans souffrir ni s'épuiser et, éventuellement, y prendre plaisir.

Il est généralement admis que l'évaluation $\mathrm{du}$ bien-être doit se faire selon des critères scientifiquement établis. Cette évaluation nécessite la mesure de caractères zootechniques, physiologiques et comportementaux. Ceux-ci incluent la mortalité, la morbidité, l'état physique et sanitaire, la capacité à exprimer des comportements spécifiques (incluant les interactions sociales, l'exploration, le jeu), l'absence d'expression de comportement aberrant et d'indication physiologique de stress. Ces notions sont celles qui sont maintenant retenues lors de l'élaboration des textes réglementaires concernant l'élevage des animaux domestiques.

\section{2 / La législation et les ins- tances réglementaires françaises}

La première loi française de protection des animaux, dite "loi Grammont", a été votée en 1850, à l'instigation du Comte de Grammont qui n'est autre que le fondateur de la Société Protectrice des Animaux (SPA). Cette loi sanctionnait les mauvais traitements infligés aux animaux domestiques, mais seulement lorsqu'ils l'étaient en public. De nombreux décrets, arrêtés ou notes de service qu'il serait par trop fastidieux de lister ici sont depuis lors venus la compléter (Fabre 1995).

Les instances réglementaires françaises compétentes sont les services vétérinaires du ministère de l'Agriculture et de la Pêche qui comprennent une administration centrale, la direction générale de l'alimentation (DGAL), et des services vétérinaires départementaux (DSV) qui sont placés sous la tutelle conjointe de la DGAL et du préfet. Au sein de la DGAL, le bureau "Protection animale et lutte contre la rage" élabore et négocie les textes européens, transcrit ces textes en droit national, informe ses interlocuteurs (professionnels, services vétérinaires départementaux, etc.). Ces textes doivent respecter les textes réglementaires européens et ne peuvent donc être moins restrictifs. Ce bureau finance également des actions de recherches ciblées en matière de protection animale. Localement, les vétérinaires inspecteurs et les techniciens des services vétérinaires départementaux sont, quant à eux, chargés de constater les infractions aux dispositions en vigueur et de dresser le cas échéant un procès-verbal. Ce procès-verbal sera ensuite transmis au procureur de la République qui jugera s'il convient de donner suite au dossier. Nous retiendrons qu'en droit pénal français, deux catégories d'infractions sont distinguées : le mauvais traitement et l'acte de cruauté. Le mauvais traitement est passible d'une peine contraventionnelle (amende, Art $\mathrm{R}$ 654-1, Code Pénal) alors que l'acte de cruauté relève d'une peine correctionnelle (amende et 6 mois d'emprisonnement, Art R 521-1, Code Pénal).

\section{3 / Les instances internationales}

Les instances internationales en matière de réglementation relative au bien-être des animaux dans les élevages consistent essentiellement en deux instances euro-

Figure 1. Mode de fonctionnement et interactions des instances europérennes en charge de la protection animale (adapté d'après Fabre 1995).

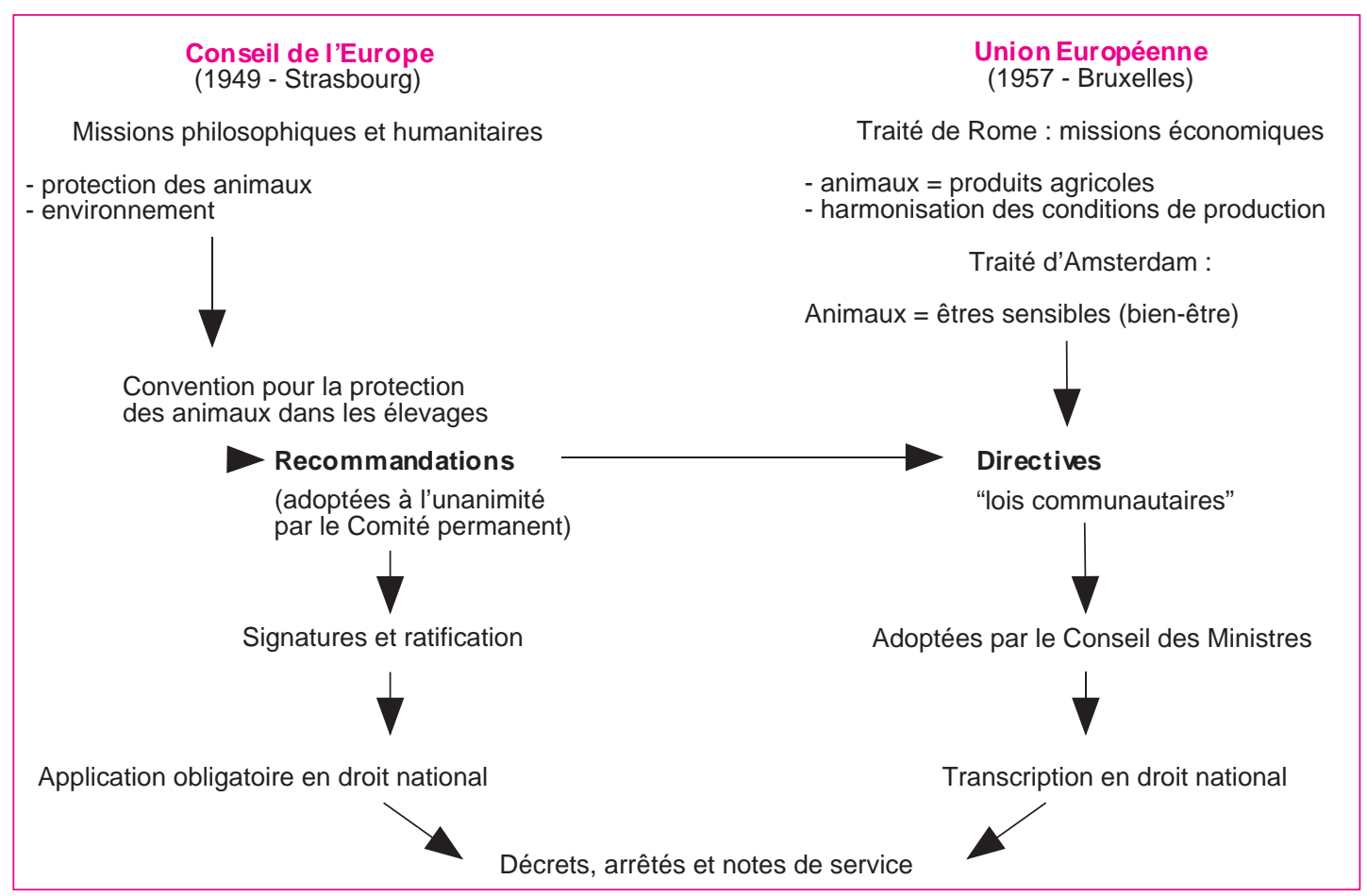


Figure 2. Processus d'élaboration d'une directive européenne - Union Européenne (adapté d'après Fabre 1995).

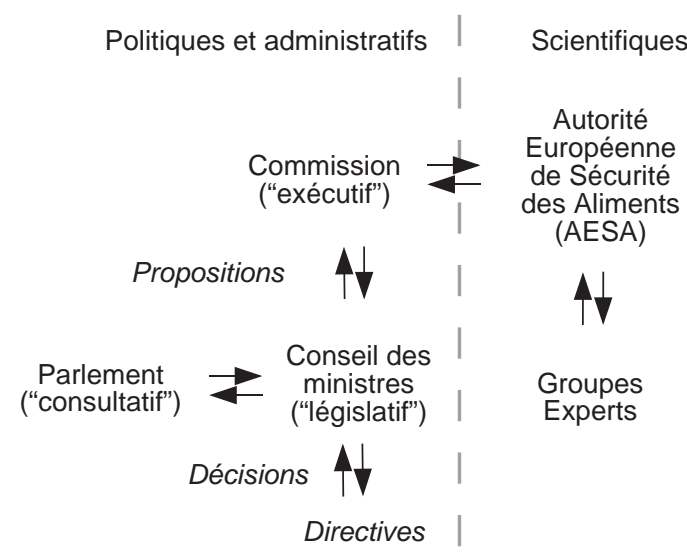

péennes, généralement confondues, l'Union Européenne (UE) et le Conseil de l'Europe (figure 1), auxquels il faut maintenant adjoindre l'Organisation Internationale des Epizooties (OIE).

L'Union Européenne, l'instance la plus connue du grand public, résulte de la création de la Communauté Économique Européenne (CEE) ou Marché Commun après adoption du Traité de Rome en 1957. Son siège est situé à Bruxelles et, après son élargissement à compter du 1er mai 2004, regroupera 25 pays membres au lieu de 15 actuellement. Le développement de l'agriculture fait partie des objectifs du Traité de Rome et les animaux y sont considérés comme des produits agricoles. La protection animale n'est pas mentionnée explicitement dans le traité de Rome, mais relève de fait de la compétence de l'Union Européenne dans la mesure où l'un de ses objectifs est d'harmoniser les conditions de production et d'éviter les distorsions de concurrence entre les états membres. Le bien-être est en outre maintenant expressément pris en considération dans la législation de l'UE, puisqu'il est précisé dans le traité d'Amsterdam (1997) que les animaux sont des êtres sensibles et que la Communauté Européenne et ses états membres doivent porter la plus grande attention au bien-être des animaux.

L'Union Européenne charge son exécutif, la Commission Européenne, d'élaborer des directives et règlements adoptables à la majorité qualifiée. Ils sont applicables dans chaque état membre après transcription en droit national, sous la forme de décrets, d'arrêtés ou encore de notes de service en France. Initiée et mise en ouvre par la commission, l'élaboration d'une directive est un processus complexe impliquant successivement ou alternativement des politiques, des administratifs et des experts scientifiques (figure 2). La version finale de ces textes est adoptée par le conseil des ministres. Le Parlement Européen n'a qu'un rôle consultatif dans le processus, mais peut à ce titre proposer des amendements. Dans ce contexte, il est important de signaler qu'un intergroupe parlementaire regroupant les nombreux députés sensibles aux aspects de protection animale a été constitué et qu'une association de protection animale européenne, Eurogroup for Animal Welfare, en assure le secrétariat. Des directives spécifiques ont déjà été adoptées pour les poules pondeuses, les porcs et les veaux, et une directive généraliste, établissant des normes minimales relatives à la protection des animaux dans les élevages, a été adoptée en 1998 (98/58, 20/07/98), sans oublier celles qui sont relatives au transport et à l'abattage des différentes espèces. Parallèlement, la commission de l'U.E. a sollicité le Comité Scientifique Vétérinaire (CSV), remplacé maintenant par l'Autorité Européenne de Sécurité des Aliments (AESA), pour que des groupes d'experts scientifiques rédigent des rapports concernant respectivement la poule pondeuse (Comité Scientifique Vétérinaire 1992 et 1996), la production de foie gras (Comité Scientifique Vétérinaire 1998) et les poulets de chair (Comité Scientifique Vétérinaire 2000). Des rapports concernant le transport des animaux et la poule pondeuse sont actuellement en cours de rédaction. Ces rapports sont destinés à faire le point de l'existant sur un thème particulier et anticipent normalement la rédaction ou le réexamen des textes réglementaires.

Le Conseil de l'Europe ne doit pas être confondu avec l'Union Européenne. En effet, bien que les 15 états membres de l'UE et l'UE en fassent partie, il s'agit d'organisations tout à fait distinctes tant en ce qui concerne leur composition que leurs objectifs. Le Conseil de l'Europe est une organisation intergouvernementale qui a été créée le 5 mai 1949. Son siège, le Palais de l'Europe, est localisé à Strasbourg. Il compte aujourd'hui 45 pays membres ainsi que quelques pays observateurs ou invités spéciaux (tableau 1). Seuls 26 pays, dont les 15 pays de l'UE, et une organisation, l'Union Européenne, ont toutefois ratifié la convention relative à la protection animale à ce jour et ont par conséquent droit de vote, mais aussi obligation de mettre en application les textes adoptés (cf tableau 1). Ce sera également le cas pour les nouveaux pays membre de l'Union Européenne à compter du ler mai 2004, s'ils ne l'ont déjà fait auparavant. À l'exception notable des problèmes de défense, le Conseil est habilité à traiter de toutes les grandes questions de la société européenne y compris les aspects éthiques et "humanitaires" liés à l'élevage des animaux domestiques. Le conseil des ministres, composée des ministres ou de leurs représentants permanents, est l'instance de décision du Conseil de l'Europe. Ses décisions, élaborées par des experts gouvernementaux, sont transmises aux gouvernements sous la forme de conventions et de recommandations dont l'adoption doit être prise à l'unanimité (cf figure 1). Les conventions ont un caractère d'obligation dans la mesure où elles s'appliquent au niveau national dès qu'un état membre les ratifie. Les recommandations s'inscrivent ensuite dans le cadre de ces conventions. Une convention relative à la protection des animaux dans les élevages a été signée en 1976, ratifiée par la France en 1978 et incluse dans notre droit 
national par le décret du 1er octobre 1980. L'application de cette convention est progressivement précisée par des recommandations pour les différentes espèces. Un comité permanent, composé d'experts provenant des administrations des différents pays contractants, est chargé de travailler sur les projets de recommandations. $Y$ siègent également des observateurs représentant différents pays (ex. États-Unis), les associations de protection animale (Eurogroup for Animal Welfare et World Society for the Protection of Animals), la profession vétérinaire (Fédération Vétérinaire Européenne), les scientifiques (International Society for Applied Ethology - ISAE) et les professionnels de l'élevage (Confédération Européenne des Agriculteurs). Ces observateurs n'ont pas

Tableau 1. Liste des pays membres du Conseil de l'Europe, du Comité permanent de la Convention Européenne sur la protection des animaux dans les élevages (pays ayant ratifié la convention STE $N^{\circ}$ 087 ; http://conventions.coe.int/Treaty) et de l'Union Européenne.

\begin{tabular}{|c|c|c|c|c|}
\hline \multirow[t]{2}{*}{$\begin{array}{l}\text { Pays membres du Conseil } \\
\text { de l'Europe }\end{array}$} & \multicolumn{3}{|c|}{$\begin{array}{l}\text { Convention Européenne sur la protection } \\
\text { des animaux dans les élevages. Date de : }\end{array}$} & \multirow{2}{*}{$\begin{array}{c}\text { Pays membres } \\
\text { de l'Union } \\
\text { Européenne }\end{array}$} \\
\hline & Signature & Ratification & Entrée en vigueur & \\
\hline \multicolumn{5}{|l|}{ Albanie } \\
\hline Allemagne & $23 / 07 / 76$ & $09 / 03 / 78$ & $10 / 09 / 78$ & $\mathrm{X}$ \\
\hline \multicolumn{5}{|l|}{ Andorre } \\
\hline \multicolumn{5}{|l|}{ Arménie } \\
\hline Autriche & $23 / 01 / 92$ & $22 / 12 / 92$ & $23 / 06 / 93$ & $\mathrm{X}$ \\
\hline \multicolumn{5}{|l|}{ Azerbaïdjan } \\
\hline Belgique & $30 / 04 / 76$ & $13 / 09 / 79$ & $14 / 03 / 80$ & $X$ \\
\hline Bosnie-Herzégovine & & $29 / 12 / 94$ & $30 / 06 / 95$ & \\
\hline \multicolumn{5}{|l|}{ Bulgarie } \\
\hline Chypre & $08 / 11 / 76$ & $15 / 04 / 77$ & $10 / 09 / 78$ & $X(01 / 05 / 04)$ \\
\hline Croatie & & $14 / 09 / 94$ & $15 / 03 / 95$ & \\
\hline Danemark & $10 / 03 / 76$ & $28 / 01 / 80$ & $29 / 07 / 80$ & $\mathrm{X}$ \\
\hline Espagne & $08 / 11 / 85$ & $05 / 05 / 88$ & $06 / 11 / 88$ & $\mathrm{X}$ \\
\hline Estonie & & & & $X(01 / 05 / 04)$ \\
\hline Finlande & $02 / 12 / 91$ & $02 / 12 / 91$ & $03 / 06 / 92$ & $\mathrm{X}$ \\
\hline France & $03 / 07 / 76$ & $10 / 01 / 78$ & $10 / 09 / 78$ & $\mathrm{X}$ \\
\hline \multicolumn{5}{|l|}{ Géorgie } \\
\hline Grèce & $30 / 04 / 76$ & $12 / 11 / 84$ & $13 / 05 / 85$ & $\mathrm{X}$ \\
\hline Hongrie & $09 / 12 / 98$ & & & $X(01 / 05 / 04)$ \\
\hline Irlande & $28 / 06 / 78$ & $07 / 04 / 86$ & $08 / 10 / 86$ & $\mathrm{X}$ \\
\hline Islande & $27 / 01 / 77$ & $19 / 09 / 89$ & $20 / 03 / 90$ & \\
\hline Italie & $23 / 04 / 80$ & $07 / 02 / 86$ & $08 / 08 / 86$ & $\mathrm{X}$ \\
\hline Lettonie & & & & $X(01 / 05 / 04)$ \\
\hline \multicolumn{5}{|l|}{ Liechtenstein } \\
\hline Lituanie & & & & $X(01 / 05 / 04)$ \\
\hline Luxembourg & $08 / 04 / 76$ & $19 / 01 / 79$ & $20 / 07 / 79$ & $\mathrm{X}$ \\
\hline Macédoine & & $30 / 03 / 94$ & $01 / 10 / 94$ & \\
\hline Malte & $29 / 09 / 88$ & 26/03/91 & $27 / 09 / 91$ & $X(01 / 05 / 04)$ \\
\hline \multicolumn{5}{|l|}{ Moldavie } \\
\hline Norvège & $28 / 01 / 80$ & $25 / 02 / 80$ & $26 / 08 / 80$ & \\
\hline Pays-Bas & $04 / 09 / 80$ & $21 / 04 / 81$ & $22 / 10 / 81$ & $\mathrm{X}$ \\
\hline Pologne & & & & $\mathrm{X}(01 / 05 / 04)$ \\
\hline Portugal & $20 / 11 / 79$ & $20 / 04 / 82$ & $21 / 10 / 82$ & $\mathrm{X}$ \\
\hline République Tchèque & $24 / 06 / 98$ & $23 / 09 / 98$ & $24 / 03 / 99$ & $X(01 / 05 / 04)$ \\
\hline \multicolumn{5}{|l|}{ Roumanie } \\
\hline Royaume-Uni & $10 / 03 / 76$ & $08 / 01 / 79$ & $09 / 07 / 79$ & $\mathrm{X}$ \\
\hline \multicolumn{5}{|l|}{ Russie } \\
\hline \multicolumn{5}{|l|}{ Saint Marin } \\
\hline Serbie Monténégro & & $28 / 02 / 01$ & $29 / 08 / 01$ & \\
\hline Slovaquie & & & & $\mathrm{X}(01 / 05 / 04)$ \\
\hline Slovénie & & $20 / 10 / 92$ & $21 / 04 / 93$ & $\mathrm{X}(01 / 05 / 04)$ \\
\hline Suède & $08 / 06 / 76$ & $07 / 12 / 77$ & $10 / 09 / 78$ & $\mathrm{X}$ \\
\hline Suisse & $07 / 07 / 76$ & $24 / 09 / 80$ & $25 / 03 / 81$ & \\
\hline \multicolumn{5}{|l|}{ Turquie } \\
\hline \multicolumn{5}{|l|}{ Ukraine } \\
\hline $\begin{array}{l}\text { Organisation Communauté } \\
\text { Européenne }\end{array}$ & $18 / 10 / 88$ & $18 / 10 / 88$ & $19 / 04 / 89$ & \\
\hline
\end{tabular}


droit de vote, mais peuvent tout de même jouer un rôle prépondérant, voire déterminant, lors de l'élaboration des textes.

Bien que ces deux institutions soient distinctes, leur fonctionnement n'est pas indépendant. Ainsi l'UE est représentée en tant que membre au sein du comité permanent du Conseil de l'Europe et, de fait, son représentant a la possibilité de voter pour l'ensemble des pays membres de l'UE (notion de vote bloqué). Cette procédure permet d'obtenir une position unanime des états membre de l'UE, au sein du comité permanent du Conseil de l'Europe, alors qu'elle a été acquise à la majorité qualifiée à Bruxelles. Parallèlement, toutes les recommandations adoptées à Strasbourg doivent être reconsidérées à Bruxelles et transcrites sous la forme de directives avec une formulation généralement plus normative et donc souvent plus contraignante.

Une troisième organisation internationale a récemment élargi son champ de compétences au bien-être animal. Il s'agit de l'Organisation Internationale des Epizooties (OIE) qui a été créée en 1924, est basée à Paris et regroupe actuellement 164 pays. Des commissions, impliquant des scientifiques, élaborent des résolutions qui sont adoptées par cette organisation et reconnues par l'Organisation Mondiale du Commerce (OMC). Le bien-être animal pourrait de ce fait être pris en considération dans le contexte des négociations internationales et les distorsions de concurrence potentiellement limitées. Un premier rapport concernant le transport des animaux est en cours de rédaction et pourrait être adopté pour 2005. L'OIE a aussi organisé en février 2004 la première conférence globale relative au bien-être animal.

\section{4 / Textes européens relatifs aux productions avicoles}

Les textes actuels relatifs aux productions avicoles s'inscrivent dans le cadre de la convention européenne relative à la "Protection des animaux dans les élevages" et une directive communautaire généraliste (UE). Cette convention, adoptée par le Conseil de l'Europe et ratifiée par la CEE en 1978, constituait jusqu'à très récemment l'unique référence en termes de droit européen. La directive communautaire généraliste (98/58/CE), l'équivalent de la convention au niveau de l'UE, a été adoptée lors du conseil du 20 juillet 1998. Les contenus de ces textes ne comportent que des dispositions générales, peu contraignantes. Ils prévoient que tout animal bénéficie d'un hébergement adapté, d'une alimentation adéquate à tout moment et de soins appropriés à ses besoins physiologiques et comportementaux en accord avec l'expérience acquise et les connaissances scientifiques. La rédaction très peu normative fait que tout élevage peut être pris en défaut au motif qu'il n'applique pas strictement l'une des obligations listées.

Concernant les productions avicoles, certains points critiques évoqués sont communs à plusieurs textes et ceux-ci peuvent globalement êtres classés en quatre catégories :

- Environnement physique et social : hébergement (bâtiment ouvert, claustration totale, cages en batteries), nature du sol (litière, caillebotis), enrichissement du milieu, densité, éclairement (naturel/artificiel, intensité lumineuse, pénombre, photopériodes fractionnées, rythmes ahémeraux), liberté de mouvement et possibilité d'avoir des interactions sociales normales.

- Conduite et pratiques d'élevage : méthodes de manipulation des oiseaux, insémination artificielle, mutilations (épointage du bec, désonglage, etc...), conditions de collecte, transport et abattage.

- Génotype : origine génétique, dimorphisme sexuel, poids vifs, inadaptations comportementales et physiologiques.

- Nutrition - alimentation : qualité et disponibilité des aliments et de l'eau, utilisation d'additifs alimentaires, de traitements vétérinaires systématiques et de promoteurs de croissance.

Les textes spécifiques et les conséquences éventuelles de leur application sont évoqués ci-après.

\section{1 / Production d'œuf}

La production d'œuf de poule pour la consommation est actuellement soumise à une nouvelle directive $(1999 / 74 / \mathrm{CE})$ qui a modifié la précédente (88/166/CEE). En outre, la recommandation concernant l'espèce Gallus gallus et adoptée en 1995, s'applique également à cette production.

Actuellement, plus de $85 \%$ de la production française d'œufs est issu de poules élevées en cages standards (photo 1). Ce mode d'élevage sera toutefois totalement interdit à compter du $1^{\text {er }}$ janvier 2012 , pour tous les élevages de plus de 350 poules. Sauf modification de cette directive lors de sa révision programmée pour 2005, les modes d'élevages alors utili-

Photo 1. Elevage de poules pondeuses en cages standard. Cliché Filières avicoles.

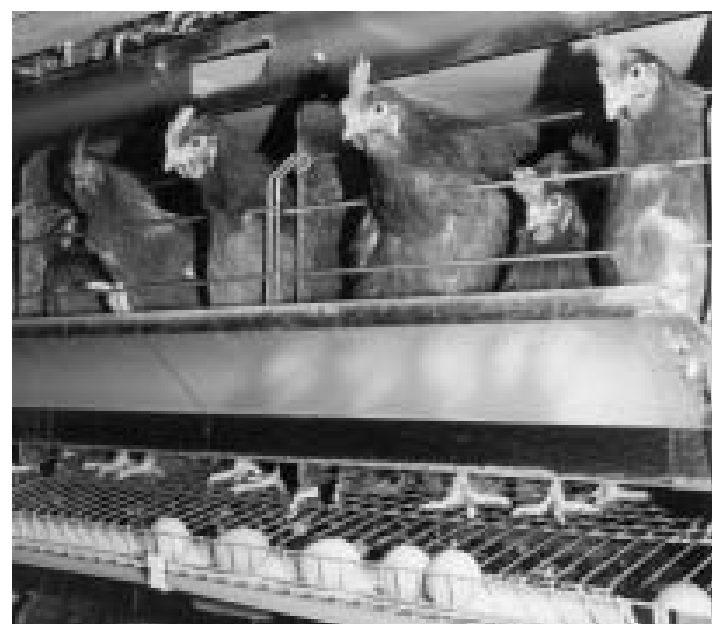


Photo 2. Eléments d'aménagement des cages pour poules pondeuses: le nid et les perchoirs. Cliché D. Guémené, INRA.

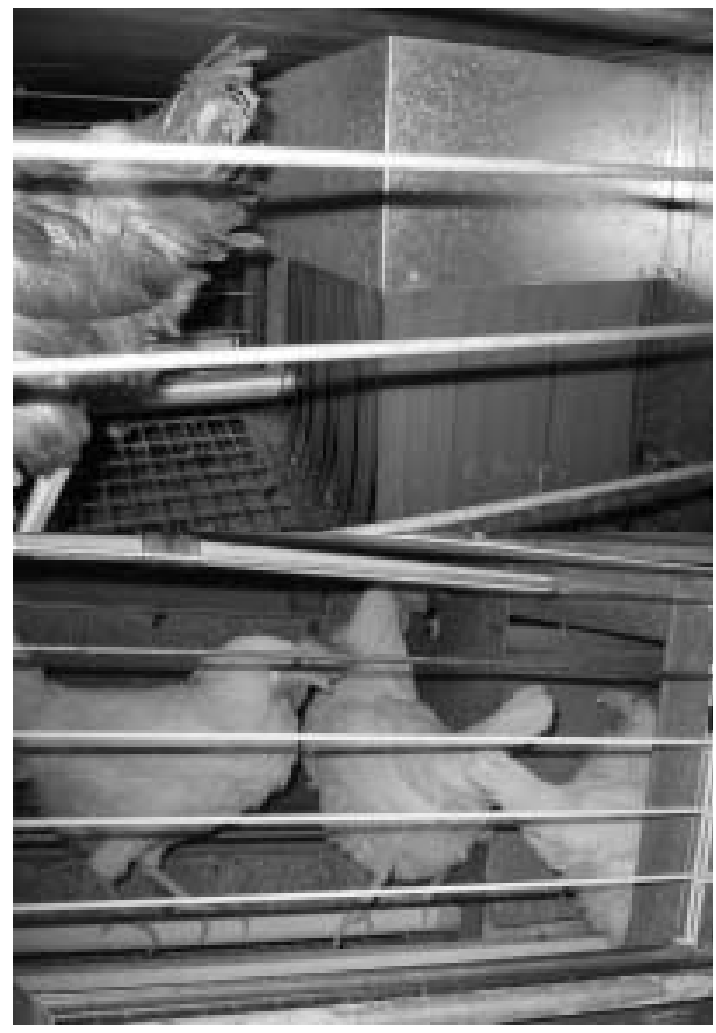

Tableau 3. Conditions de production d'œufs pour des poules placées en volières ou au sol depuis le $1^{\text {er }}$ janvier 2002, en application de la directive (99/74/CE).

\begin{tabular}{|l|c|c|}
\hline & Volière & Sol \\
\hline Densité (poule/m²) & $9 / 12$ & 9 \\
\hline $\begin{array}{l}\text { Surface litière } \\
\left(\mathrm{cm}^{2} / \text { poule) }\right.\end{array}$ & & $\begin{array}{c}\geq 250 \\
(1 / 3 \text { surface })\end{array}$ \\
\hline $\begin{array}{l}\text { Mangeoire } \\
\text { (cm/poule) }\end{array}$ & $\begin{array}{c}10 \\
(4 \text { circulaire })\end{array}$ & $\begin{array}{c}10 \\
(4 \text { circulaire })\end{array}$ \\
\hline $\begin{array}{l}\text { Perchoirs } \\
(\mathrm{cm} / \text { poule) }\end{array}$ & 15 & 15 \\
\hline $\begin{array}{l}\text { Nid (nb/poule) ou } \\
\left(\mathrm{m}^{2} / \mathrm{nb} \text { poule) }\right.\end{array}$ & $\begin{array}{c}1 / 7 \text { ou } \\
1 \mathrm{~m}^{2} / 120 \\
\text { poules }\end{array}$ & $\begin{array}{c}1 / 7 \text { ou } \\
1 \mathrm{~m}^{2} / 120 \\
\text { poules }\end{array}$ \\
\hline
\end{tabular}

sables seront la cage aménagée, la volière et les divers systèmes de production au sol avec accès ou non à un parcours extérieur (tableaux 2 et 3). Concernant la cage aménagée, sa surface au sol devra être d'au moins $2000 \mathrm{~cm}^{2}$, d'une hauteur minimale de $45 \mathrm{~cm}$ et être équipé de nids, de dispositifs de raccourcissement des griffes, de perchoirs d'une longueur de $15 \mathrm{~cm}$ par poule (photo 2) et d'un bac contenant des matériaux friables permettant l'expression du comportement de bain de poussières et pour ce motif communément appelé bac à poussière. La surface utile, c'està-dire d'une hauteur minimale de $45 \mathrm{~cm}$ et de dimensions sur tous cotés aux moins égales à $20 \mathrm{~cm}$, devra être de $600 \mathrm{~cm}^{2}$ minimum par poule; surface à laquelle il faut adjoindre une zone complémentaire pour porter la surface totale par poule à $750 \mathrm{~cm}^{2}$. La hauteur de cette zone complémentaire doit quant à elle être au minimum de $20 \mathrm{~cm}$ et peut par exemple correspondre à l'espace réservé au nid et au bac à poussière $\left(75 \times 2=150 \mathrm{~cm}^{2}\right)$. À titre de comparaison, la société MacDonald a récemment exigé de ses fournisseurs aux USA que la surface par poule soit portée de 300 à $460 \mathrm{~cm}^{2}$. Chaque poule doit par ailleurs disposer d'une longueur de $12 \mathrm{~cm}$ de mangeoire et d'un minimum de deux abreuvoirs, comme un système de pipettes goutte-à-goutte avec ou sans coupelles, doit être accessible à tout moment. Actuellement, divers prototypes de cages aménagées sont en cours d'évaluation scientifique même si cette réglementation s'applique déjà pour toutes les nouvelles installations. En outre, pour les installations actuelles, la directive impose, depuis le $1^{\text {er }}$ janvier 2003, que chaque poule logée dans une cage dispose d'une surface minimale de $550 \mathrm{~cm}^{2}$ contre $450 \mathrm{~cm}^{2}$ auparavant (tableau 2). Pour les volières et les systèmes d'élevage au sol (photo 3), la législation est entrée en vigueur depuis le $1^{\text {er }}$ janvier 2002 pour les nouvelles installations et sera applicable à compter du $1^{\text {er }}$ janvier 2007 ou 2012 pour toutes les installations existantes (tableau 3). La densité ne doit pas y excéder 9 poules $/ \mathrm{m}^{2}$ de surface utilisable, toutefois elle peut être maintenu à 12 poules/m² jusqu'au 31 décembre 2011 tant que la surface utilisable correspond à la surface totale au sol. La zone couverte de litière doit être au moins de $250 \mathrm{~cm}^{2}$ par poule et représente au moins 1/3 de la surface totale. La longueur des mangeoires longitudinales doit être de $10 \mathrm{~cm}$ par poule et de $4 \mathrm{~cm}$ pour les man-

Tableau 2. Conditions d'utilisation des cages standard et aménagées pour la production d'œufs de poule, en application de la directive (99/74/CE).

\begin{tabular}{|l|c|c|c|}
\hline & $\begin{array}{c}\text { Cage } \\
\text { Avant le 01/01/2003 }\end{array}$ & $\begin{array}{c}\text { standard } \\
\text { Depuis le 01/01/2003 }\end{array}$ & $\begin{array}{c}\text { Cage aménagée } \\
\text { Depuis le 01/01/2003 }\end{array}$ \\
\hline $\begin{array}{l}\text { Surface utile }\left(\mathrm{cm}^{2} / \text { poule) }\right. \\
\text { Surface autre }\left(\mathrm{cm}^{2} / \text { poule) }\right.\end{array}$ & 450 & 550 & $\begin{array}{c}\geq 600 \\
\text { Surface totale }\left(\mathrm{cm}^{2}\right)\end{array}$ \\
\hline Hauteur $(\mathrm{cm})$ & 40 & $4020 \mathrm{~cm}$ haut. $)$ \\
Mangeoire $(\mathrm{cm} /$ poule $)$ & 10 & 10 & 45 \\
Perchoirs (cm/poule) & & & 12 \\
\hline Nid & & & 15 \\
Bac à matériaux friables & & & oui \\
"Racourcisseur" de griffes & & & oui \\
oui
\end{tabular}

(1) La cage standard sera interdite à compter du $1^{\text {er }}$ janvier 2012. 
geoires circulaires. La longueur de perchoir doit être de $15 \mathrm{~cm}$ par poule. On doit placer au sein du bâtiment, des nids individuels (un pour 7 poules) ou un nid collectif de $1 \mathrm{~m}^{2}$, ou plus, pour un maximum de 120 poules. Cette directive aura indéniablement un impact essentiel sur l'avenir de la production d'œeufs en l'Europe (Windhorst 2001, Wolffram et al 2002). Lune des conséquences pratiques sera l'augmentation du coût de production qui est estimée à environ $13 \%$ pour la cage aménagée et à $17 \%$ pour la volière (Gonnier 2003). Il est cependant encore difficile d'estimer précisément ce que seront les conséquences pratiques à plus longue échéance en termes de diminution de la production locale et/ou d'augmentation des importations. De surcroît, des distorsions de concurrence intracommunautaire seront importantes puisque certains pays peuvent prendre des mesures unilatérales plus restrictives ; ainsi l'Allemagne a unilatéralement décidé d'interdire l'usage des cages pour la production d'oufs sur son territoire.

\section{2 / Production de poulet de chair}

Les convention et directive généralistes ainsi qu'une recommandation spécifique (Gallus gallus) adoptée en 1995 s'appliquent à cette production. Parallèlement, la commission de l'UE a sollicité son Comité Scientifique pour la rédaction d'un rapport relatif au bien-être du poulet de chair. Dans ce rapport, les experts ont souligné les problèmes résultant de l'utilisation de génotypes à croissance rapide et leur placement à des densités élevées et suggéré des alternatives (photo 4).

Les auteurs de ce rapport ont suggéré que l'index de sélection utilisé intègre des paramètres concernant le bien-être et la santé même si ce choix réduit la pression de sélection sur le taux de croissance et l'indice de consommation. Les sélectionneurs doivent aussi êtres capables de démontrer que leurs programmes de sélection ne sont pas préjudiciables au bien-être. Ces experts indiquent qu'il est probable que le bien-être des poulets sera affecté si la densité excède $30 \mathrm{~kg} / \mathrm{m}^{2}$ et de telles conditions devront êtres évitées. En outre, la densité doit aussi être adapté aux possibilités de ventilation au sein des poulaillers. Les poulets devraient aussi êtres élevés dans des conditions permettant d'éviter le recours à des mutilations, comme l'épointage du bec ou la taille des griffes. Ainsi, il est éventuellement possible de stimuler l'expression d'une plus large gamme de comportements normaux en enrichissant le milieu d'élevage. Il est probable que certaines des suggestions faites dans ce rapport seront prises en considération pour la rédaction, en cours, de la directive concernant cette production.

\section{3 / Production de palmipèdes}

Trois recommandations concernant les palmipèdes ont été adoptées en 1999. Elles concernent respectivement l'oie domestique
Photo 3. Elevage de poules pondeuses au sol avec accès à un parcours. Cliché Filières avicoles.

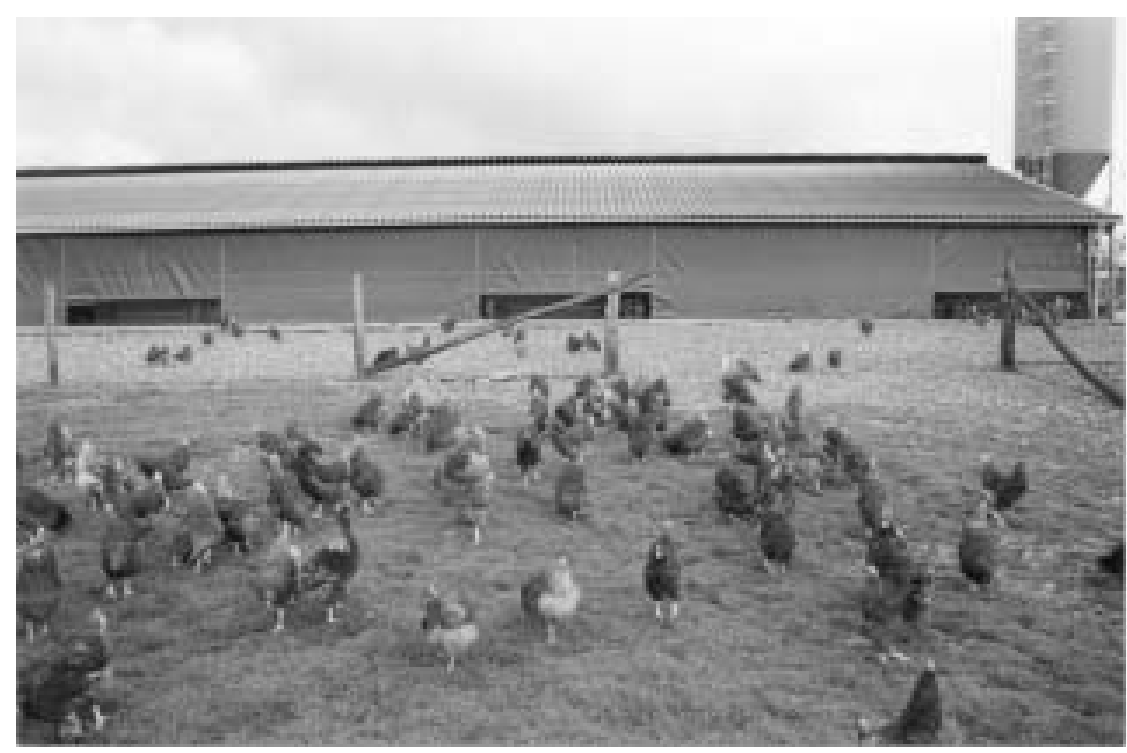

Photo 4. Elevage sur parcours de poulets de chair de génotype à croissance lente. Cliché Filières avicoles.

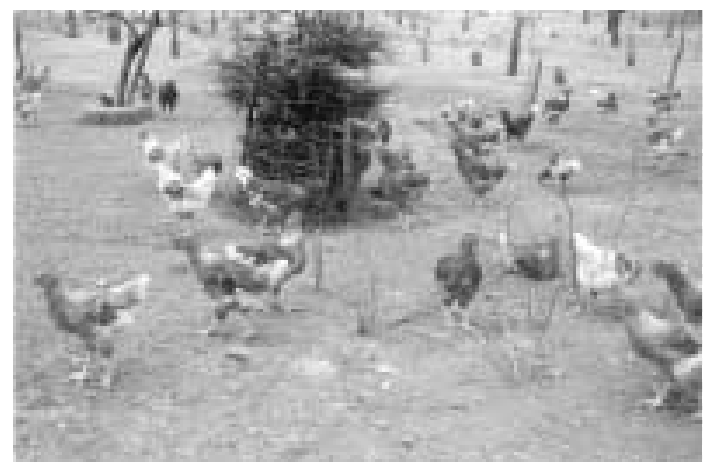

(Anser sp.) (T-AP [95/5]), le canard commun (Anas platyrhynchos) (T-AP [94/3]), le canard de barbarie (Cairina moschata) et le canard mulard (un hybride des canards de barbarie et commun) (T-AP [95/20]). Le paragraphe 3 de l'article 9 de la convention précise que ces recommandations sont entrées en vigueur depuis le 31 décembre 1999. Les recommandations s'appliquent aux nouvelles installations ou lors du remplacement des installations existantes à compter du 31 décembre 2004 et pour toutes au 31 décembre 2010 .

Nous retiendrons de ces recommandations que les sols en caillebotis intégral et les cages individuelles sont proscrits pour tous les palmipèdes (photo 5), alors que les pratiques d'épointage du bec et de désonglage ne sont plus tolérées que dans un contexte très restrictif, pour les seuls canards de barbarie et mulards. La production de duvet par collecte régulière des plumes sur animaux vivants telle quelle est encore pratiquée dans plusieurs pays, dont la Hongrie, est interdite. La production de foie gras ne pourra être poursuivie que dans les zones où elle a cours actuellement et ce, conformément à la législation en vigueur dans les pays concernés. Les méthodes de restriction alimentaire, les rythmes lumineux ahémeraux et les photopé- 
Photo 5. Elevage de canards mulards. Les sols exclusivement recouverts de caillebotis sont interdits. Ils doivent comporter une surface de repos recouverte de litière. Cliché D. Guémené, INRA.

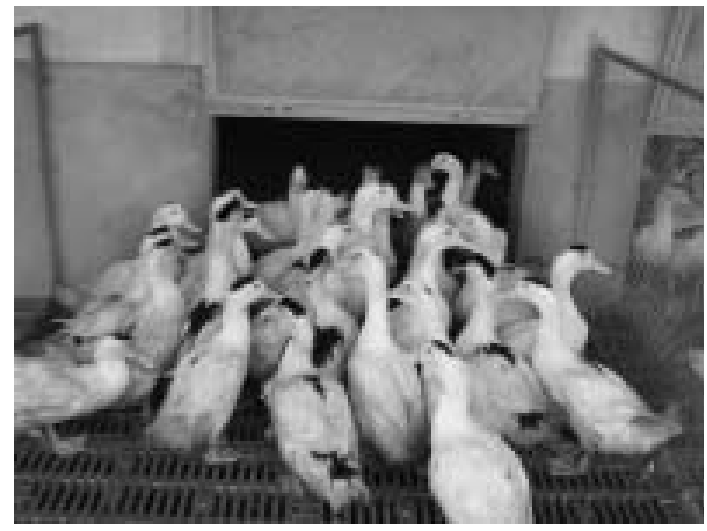

riodes fractionnées devraient êtres interdits, de même que l'utilisation de systèmes d'abreuvement de type pipette. Le transport d'oiseaux en les tenant seulement par les pattes et avec la tête pendante n'est pas toléré. Plusieurs de ces dispositions n'ont pas réellement de caractère normatif et sont donc assez peu contraignantes dans la version actuelle des textes des recommandations.

Parallèlement, la Commission Européenne a commandité un rapport concernant la production de foie gras au Comité Scientifique Vétérinaire. La conclusion générale émise par ce groupe est la suivante : "la pratique du gavage dans les conditions actuelles est préjudiciable au bien-être des oiseaux". La publication de ce document ne préjuge pas de l'adoption, dans un proche avenir, de directives relatives à ces espèces, mais cette éventualité n'est pas à exclure. Les directives sont par nature normatives et pourraient par conséquent être beaucoup plus contraignantes en termes de conduite d'élevage et pour l'éleveur. Avant cette échéance, les recommandations concernant les palmipèdes feront vraisemblablement l'objet d'une note de service de la part des autorités compétentes françaises. Certains pays dont l'Italie, qui n'en produisait pas avant, et la Pologne ont par ailleurs déjà officiellement interdit le gavage sur leur territoire. Concernant la production polonaise, elle n'était plus concurrentielle en raison du coût prohibitif d'importation du maïs.

\section{4 / Production de dinde}

La recommandation concernant l'espèce dinde (Meleagris gallopavo) fut la dernière à être adoptée pour les oiseaux (juin 2001). Son organisation générale est assez comparable à celles qui concernent les autres espèces de volailles ; toutefois, une évolution vers un contenu plus normatif est perceptible. L'utilisation de rythmes ahémeraux et de photopériodes fractionnées, qui sont des pratiques communes en France pour cette espèce, est interdite. Parallèlement, une période d'obscurité (scotopériode) continue d'une durée minimale de 4 heures est exigée.
Photo 6. Enrichissement du milieu d'élevage des dindes : plaques destinées à prévenir le picage et plateformes pour le perchage. Cliché D. Huonnic, AFSSA Ploufragan.

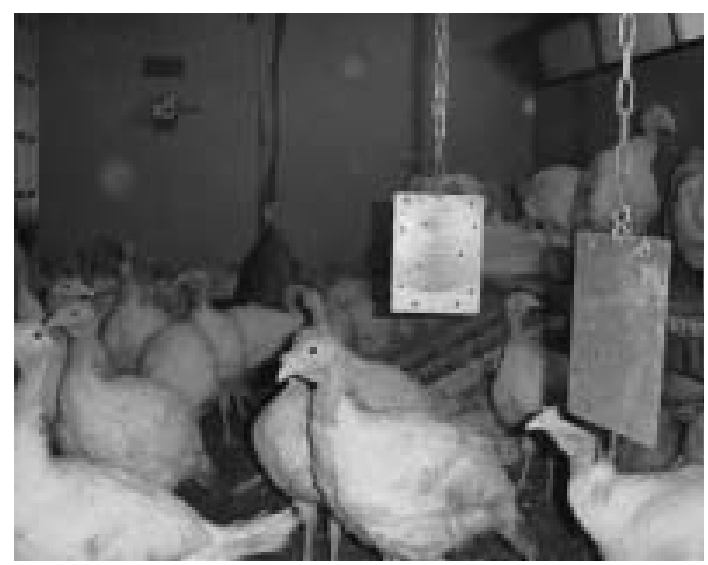

Lintensité lumineuse doit être de 10 lux minimum. Les mutilations, comme l'épointage du bec et la coupe des ongles, sont interdites à moins qu'une autorisation spécifique n'ait été sollicitée et, sous réserve d'obtention, le traitement doit alors être pratiqué avant l'âge de 10 jours. Afin de limiter le picage, il est suggéré d'enrichir le milieu d'élevage (photo 6).

\section{5 / Autres espèces d'oiseaux exploitées commercialement}

La convention pour la protection d'animaux de ferme (1976) et la directive généraliste (1998) s'appliquent à toutes les espèces animales et donc à toutes les espèces d'oiseaux exploitées commercialement. Une recommandation spécifique concernant les ratites (autruche, émeu et nandou) a été adoptée en 1997. Son contenu se réfère principalement aux conditions d'hébergement de chacune des espèces. Les instances européennes n'ont, à notre connaissance, pas encore adopté de texte pour d'autres espèces d'oiseaux qui font également l'objet d'une exploitation commerciale, comme la pintade, la caille, le pigeon, le faisan ou les perdrix. Le texte d'une recommandation concernant le faisan, même si il n'a pas été adopté à ce jour, est toutefois en cours de rédaction depuis 1994, ainsi qu'une réflexion concernant la caille. Il est donc probable que des textes seront progressivement adoptés pour ces différentes espèces.

\section{Conclusions}

La mise en place d'une réglementation européenne et nationale relative au bien-être animal intervient progressivement pour l'ensemble des filières animales. Le contenu de ces textes réglementaires doit avoir une justification scientifique ; toutefois, la littérature et les résultats scientifiques spécifiques ne sont pas toujours disponibles. En leur absence, les extrapolations entre espèces sont une pratique fréquente et des normes ou des équipements peuvent ainsi êtres préconisés avant que leur adéquation n'ait été évaluée. À ce 
stade, l'omniprésence des associations de protection animale dans l'entourage immédiat, voire au sein même, des instances chargées de l'élaboration de cette réglementation et leur propension à l'activisme via des actions de lobbying et/ou médiatiques, le financement d'actions de recherche, etc., conduit à s'interroger quant à leur impartialité et à leur degré d'implication en regard de leur représentativité réelle dans l'opinion publique. Cette situation est d'autant plus préoccupante qu'une réglementation unique s'applique pour l'ensemble des pays, alors que différents génotypes ou modes de production sont utilisés par les producteurs. Ainsi, les oiseaux peuvent êtres élevés dans des conditions tout à fait différentes (âge et poids à l'abattage, mode d'alimentation, rythmes lumineux, etc) selon le pays ou la zone géographique considéré. Parallèlement, les textes réglementaires peuvent êtres réexami- nés après leur entrée en vigueur, et, le cas échéant, amendés en fonction de toute nouvelle connaissance scientifique disponible. Cette relative instabilité induit une incertitude quant aux choix stratégiques et risque par conséquent d'être préjudiciable au développement des filières. Il convient également de préciser que cette réglementation ne s'applique pas aux pays tiers, y compris les pays d'Europe de l'Est qui sollicitent actuellement l'adhésion à l'UE et que, dans ce contexte international, les risques de distorsion de concurrence sont importants. Une délocalisation de tout ou partie de certaines productions, sans garantie du respect de conditions sanitaires et de bien-être, n'est donc pas à exclure et de fait est déjà en cours notamment pour le poulet de chair. Dans cette hypothèse, les problèmes de bien-être en élevage ne seront pas résolus mais exportés vers des pays tiers.

\section{Références}

Broom D.M., 1986. Indicators of poor welfare. Br. Vet. J., $142,524-526$

Code Rural, 1991. Livre premier, titre cinquième, article 276 et suivants. Ed. Dalloz, Paris.

Comité Permanent de la Convention Européenne sur la protection des animaux dans les élevages, 1976. European convention for the protection of animals kept for farming purposes.

Comité Permanent de la Convention Européenne sur la protection des animaux dans les élevages, 1986. Recommandations concernant les volailles de l'espèce Gallus gallus élevées pour la ponte. T-AP, 17 p.

Comité Permanent de la Convention Européenne sur la protection des animaux dans les élevages, 1997. Recommandations concernant les ratites (Autruches, Emeus, Nandous). T-AP, 94 (1), $17 \mathrm{p}$

Comité Permanent de la Convention Européenne sur la protection des animaux dans les élevages, 1999. Recommandations concernant les canards domestiques (Anas platyrhynchos). T-AP, 94 (3), $13 \mathrm{p}$.

Comité Permanent de la Convention Européenne sur la protection des animaux dans les élevages, 1999. Recommandations concernant les canards de barbarie (Cairina moschata) et les hybrides de canards de barbarie (Cairina moschata) et de canards domestiques (Anas platyrhynchos). T-AP, 95 (20), $16 \mathrm{p}$

Comité Permanent de la Convention Européenne sur la protection des animaux dans les élevages, 1999. Recommandations concernant les oies domestiques (Anser anser F. domesticus, (Anser cygnoides F. domesticus) et leurs croisements. T-AP, 95 (5), $14 \mathrm{p}$

Comité Permanent de la Convention Européenne sur la protection des animaux dans les élevages, 2001. Recommandation concernant les dindes (Meleagris gallopavo). T-AP, 95 (16).

Comité Permanent de la Convention Européenne sur la protection des animaux dans les élevages (en cours). Projet de recommandations concernant les faisans. T-AP, 94 (23), 9 p.

Dawkins M., 1983. La souffrance animale ou l'étude objective du bien-être animal. Ed. Point Vétérinaire, Paris.
Fabre A., 1995. Bien-être des animaux dans les élevages : enjeux et perspectives d'une réglementation nationale. Le Point Vétérinaire, 27 (170), 283-292.

Gonnier V., 2003. Situation et évolution des différentes filières de ponte européennes. 5ème Journées de la Recherche Avicole, Tours, 26-27 Mars 2003, 19-27.

Journal Officiel des Communautés Européennes, 1988. Directive 88/166/CEE du Conseil du 7-03-88 établissant les normes minimales relatives à la protection des poules pondeuses en batterie. JO L 74/83, 258-262.

Journal Officiel des Communautés Européennes, 1998 Directive 98/58/CE du Conseil du 20-09-98 concernant la protection des animaux dans les élevages. JO L $221 \mathrm{du}$ 8/8/98, 23-27.

Journal Officiel des Communautés Européennes, 1999. Directive 99/74/CE du Conseil du 19-07-99 concernant les volailles de l'espèce Gallus gallus élevées pour la ponte. JO L 203/53 du 3/08/99, 53-57.

Scientific Veterinary Commitee, Animal Welfare Section, 1992. Report on the welfare of laying hens kept in different production systems.

Scientific Veterinary Commitee, Animal Welfare Section, 1996. Report on the welfare of laying hens. CEC, DGAVI/BII.2.

Scientific Veterinary Commitee, Animal Welfare Section, 1998. Welfare aspects of the production of foie gras in ducks and geese. CEC, DGXXIV.

Scientific Veterinary Commitee, Animal Welfare Section, 2000. The welfare of chickens kept for meat production (broilers). CEC, DGXXIV.

Service des Relations Publiques du Conseil de l'Europe, 1996. Le Conseil de l'Europe : activités et réalisations, $67 \mathrm{p}$.

Windhorst H.W., 2001. Is there a future for Europe's egg industry ? Poultry International, 40 (7), 26-32.

Wolffram R., Simmons J., Giebel A., Bongaerts R., 2002. Impacts of stricter legal standards in the EU for keeping laying hens in battery cages. Wold's Poultry Science J., 58, $365-370$ 


\begin{abstract}
Poultry production: welfare and European legislation.

In Europe, two organisations control legislation concerning domestic animal welfare: these are the Council of Europe and the European Union. A general convention covering all domestic species (1976) and a comparable directive $(98 / 58 / \mathrm{CE}, 1998)$ have been adopted, by the standing committee of the European Convention (Council of Europe) and the European Union, respectively. Both of these texts apply to all domestic species and therefore to domestic birds. Furthermore, in 1999, the European Union adopted a second version of a specific directive concerning the laying hen (Gallus gallus). Scientific reports concer-

Authority [E.F.S.A.]) of the E.U. and a directive concerning the broiler production is under preparation. In the meantime, the standing committee of the European Council has adopted six specific recommendations devoted to laying hens (1986), ratites (1997), waterfowl (3 recommendations in 1999) and turkeys (2001). In accordance with the convention of 1976, all these recommendations should be reviewed within 5 years after their adoption and be amended if appropriate. Furthermore, according to directive 98/58/CE (European Union, 1998), each adopted recommendation should be incorporated in the law of member states and applied. In any case, these texts can only be more restrictive.
\end{abstract} ning laying hens $(1992,1996)$, "foie gras" production (1998) and broiler breeders (2000) have also been commissioned at the request of the scientific veterinary committee (presently European Food Safety
GUEMENE D., FAURE J.-M., 2004. Productions avicoles, bien-être et législation européenne. INRA Prod. Anim., 17, 59-68. 